\title{
Tapentadol extended release in the management of peripheral diabetic neuropathic pain
}

This article was published in the following Dove Press journal:

Therapeutics and Clinical Risk Management

14 January 2015

Number of times this article has been viewed

\author{
Nalini Vadivelu' \\ Alice $\mathrm{Kai}^{2}$ \\ Benjamin Maslin \\ Gopal Kodumudi ${ }^{3}$ \\ Aron Legler' \\ Jack M Berger ${ }^{4}$ \\ 'Department of Anesthesiology, \\ Yale University School of Medicine, \\ New Haven, CT, USA; ${ }^{2}$ Stony Brook \\ University School of Medicine, \\ Stony Brook, NY, USA; ${ }^{3}$ Department \\ of Structural and Cellular Biology, \\ Tulane University, New Orleans, LA, \\ USA; ${ }^{4}$ Department of Anesthesiology, \\ Keck School of Medicine, University \\ of Southern California, Los Angeles, \\ CA, USA
}

\begin{abstract}
Tapentadol, a $\mu$-opioid agonist and norepinephrine reuptake inhibitor, has been found to be an effective medication for a wide variety of chronic pain conditions, including back pain, cancer-related pain, and arthritic pain. It has also been found to have fewer gastrointestinal side effects than more traditional opioid-based therapies. More recently, tapentadol extended release has been demonstrated to be effective in the management of painful diabetic neuropathy, an often debilitating condition affecting approximately one-third of all patients with diabetes. This review highlights the most up-to-date basic and clinical studies by focusing on the mechanisms of action of tapentadol and its clinical efficacy, especially with regard to painful diabetic neuropathy.
\end{abstract}

Keywords: chronic pain, neuropathic pain, pharmacology, analgesia, pain management

\section{Introduction to the management issues associated with the painful diabetic neuropathy (PDNP) in adults PDNP}

PDNP, which affects approximately one-third of all patients with diabetes, has wide implications on the quality of life and general health of affected patients. Neuropathic pain, defined as pain caused by a lesion or disease of the somatosensory nervous system, ${ }^{1}$ is not a result of a nociceptive source or a particular stimulus. Commonly, slight injuries produce pain that is seemingly disproportionate to any potential tissue damage (hyperalgesia), and will encompass an area broader than where the insult was inflicted. ${ }^{2}$ Although the exact mechanism of neuropathic pain still has not been elucidated, it is believed that myelinated fiber density is diminished because of the release of proinflammatory cytokines in response to nerve damage. ${ }^{3}$ Recently, glial cells, including astrocytes and microglia, have been implicated in neuropathic pain because they form close connections with neurons. ${ }^{4}$ Growing evidence also supports the role of central sensitization in the pathophysiology of neuropathic pain. That is, increased sensitivity at the dorsal horn at the level of the spinal cord results from afferents acquiring synaptic efficiency, which causes lower pain thresholds and increased excitability within the ascending system of the pain pathway. ${ }^{5}$

The pathophysiology of neuropathic pain involves the restorative and inflammatory processes that are triggered once injury occurs and cause a hyperexcitable state that is associated with peripheral sensitization. In the normal host, inflammation will abate as the injury mends. However, in an altered milieu (as is observed in diabetes) or with persistent damaging triggers, nociception continues because of recurrent stimulation. Sensitization and stimulation of nociceptors, both of which can be caused by numerous factors, effectively lower neuronal thresholds of action potentials and promote
Correspondence: Nalini Vadivelu

Department of Anesthesiology Yale University School of Medicine

333 Cedar Street, TMP 3

New Haven, CT 06520, USA

Email nalini.vadivelu@yale.edu 
peripheral sensitization. These factors include inflammatory mediators that are discharged from nociceptive terminals, including substance $\mathrm{P}$ and the calcitonin gene-related peptide. These mediators promote vascular permeability, which causes local edema and the leakage of growth factors, prostaglandins, cytokines, and bradykinin. It is thought that the multifactorial dependence of various substances on producing nociceptive sensitization may explain why there is no single comprehensively effective drug. ${ }^{6}$

Hyperalgesia, or the perception of pain which is disproportionate to the damage sustained by the peripheral pain fibers, is classified as either primary or secondary. Primary hyperalgesia accompanies injury to tissue as an outcome of peripheral nociceptor sensitization. In contrast, secondary hyperalgesia is found in the neighboring intact tissue because of the sensitization of the central nervous system. It is thought that this sensation can be attributed to either the extension of the receptive fields of damaged nerves, or by transmission of excitatory signals that spread through neighboring uninjured nerve fibers (ie, ephaptic transmission). ${ }^{6}$

Discharges of ectopic origin can lead to perceived pain that is spontaneous in nature. This pain can stem from undamaged neighboring fibers, the dorsal root ganglion, or along other points of the damaged nerve. ${ }^{7}$ Allodynia, or perceived pain that is produced by a stimulus that is normally not painful, may result from reduced neuronal thresholds and can be characterized as either thermal or mechanical in nature. ${ }^{6}$

Within the ascending track in the sensory nervous system, nociceptive signals are transmitted as action potentials from $\mathrm{A} \delta$ and $\mathrm{C}$ nociceptors to second and third order neurons situated in the spinal cord's dorsal horn. These neural signals are crucial in forming the excitatory signal that follows any noxious stimulus. They are transmitted to portions of the brain that are responsible for processing the sensory components of pain. Additionally, there are corresponding projections to limbic regions of the brain that are involved in processing these signals to generate the perceived element of pain. ${ }^{5}$

The descending pathway consists of the response signals traveling to the dorsal horn of the spinal cord via the rostral ventromedial medulla and the periaqueductal grey of the midbrain. In the dorsal horn, endogenous ligands, including serotonin, noradrenaline, and other neurotransmitters, are released and are bound by presynaptic receptors located on primary afferent fibers and are bound to postsynaptic receptors located on transmission neurons of the spinal cord. These receptors function to mediate and regulate the influx of transmittance of noxious signals. This top-down transmission pathway allows the brain to regulate signals of pain at anatomically lower (spinal) levels. ${ }^{5}$ Noxious stimuli that are processed by the limbic system result in either the excitation or the inhibition of the processing of the pain through the "on" or "off" cells within the portion of the descending pathway constituted by the rostral ventromedial medulla. ${ }^{8}$

\section{Clinical presentation of PDNP}

Patients with PDNP often present with symmetrical pain in the lower extremities that progresses proximally. The pain is unprovoked by any external stimulus and is described as a painful numbness, burning, or a stabbing sensation in the "stocking and glove" distribution. ${ }^{9}$ In an Israeli study, the predominant locations of pain were the head (13\%), the limbs (17\%), and the back (32\%). ${ }^{10}$ Of the patients with neuropathic pain associated with type II diabetes mellitus, $73.6 \%$ described having pain for longer than a year. These patients also recounted that the nature of the pain was continuous, would worsen in the evenings, and would affect day-to-day activities and mood. Of these patients, $61.4 \%$ described a tingling or stabbing of the skin that accompanied the pain, and $55 \%$ experienced numbness or were hypersensitive to stimuli. ${ }^{11}$

PDNP is often categorized into three discrete categories: muscular pain, dysesthetic pain, and paresthetic pain. Muscular pain is often described as one that is deep and dull and stems from the neuropathy of large fibers. In contrast, dysesthetic and paresthetic pains derive from neuropathy of small fibers. Dysesthetic pain is often perceived as a prickling or burning sensation, whereas paresthetic pain is sensed as "pins and needles". ${ }^{12}$

\section{Challenges in the management of PDNP}

The lack of effective treatments to manage PDNP contributes to its high burden, especially among patients with chronic pain. This can be contrasted with the numerous effective treatment modalities used to manage nociceptive pain. In general, adjuvants that are used to manage neuropathic pain only have a minimal therapeutic effect and are only beneficial in only a small portion of affected patients. The underlying problem is the lack of understanding of the exact pathological mechanisms associated with neuropathic pain. ${ }^{13}$

Peripheral neuropathy is a complex disease process that is often associated with conditions that promote further damage to nerve endings, such as chemotherapy, zoster infection, thiamine and $\mathrm{B}_{12}$ deficiencies, HIV, hypothyroidism, systemic lupus erythematosus, Lyme disease, and, of course, diabetes. ${ }^{9}$ In addition, it is important to note that diabetic neuropathy affects not only sensory nerves, which 
has implications for PDNP, but affects a wide variety of components of the nervous system. Motor nerves, including cranial nerves and autonomic nerves, can be affected as well. ${ }^{14}$ Other conditions in which neuropathic pain persists include radiating lower back pain and postherpetic neuralgia. Currently, the medications available for targeting such pain include gabapentin, pregabalin, mixed serotonin and noradrenaline reuptake inhibitors, anticonvulsants, tricyclic antidepressants, and opioids. Unfortunately, many of these treatment options are conservatively prescribed and have restricted effectiveness because of severe side effects at the necessary dosages. ${ }^{5}$

One of the most frequent side effects accompanying longterm opioid treatment is constipation, ${ }^{15}$ which is prone to be more persistent with long-term treatment. ${ }^{16-19}$ Opioid usage is also often accompanied by various gastrointestinal side effects, including nausea and vomiting that is usually transitory. Two US surveys of patients undergoing opioid treatment for pain (both acute and chronic) demonstrated that greater than $50 \%$ of patients experienced constipation. ${ }^{20}$ Difficulties in the management of pain arise when prescribed opioids produce side effects that are best addressed by reducing dosages to substandard levels or by terminating therapy with the drug. ${ }^{19}$

Within the first week of opioid therapy, opioid-related vomiting and nausea usually stops because enough tolerance has been built, ${ }^{21}$ but constipation seldom improves with prolonged opioid use. ${ }^{22}$ Furthermore, regular prophylactic laxative therapy does not effectively relieve opioid-usage induced constipation. ${ }^{23-26}$ In one survey of patients taking concomitant daily laxatives and opioids, $33 \%$ of the patients reported decreasing dosages, missing doses, or stopping the consumption of opioids to mitigate opioid-induced constipation. $^{23}$

There are three types of opiate receptors that have been acknowledged to affect gastrointestinal functions in humans. These receptors are classified as G-protein-coupled receptors that work via inhibition of adenylate cyclase and thereby effectively diminish the release of acetylcholine and neuronal excitability. These receptors include: $\mu$-receptors, $\delta$-receptors, and $\kappa$-receptors, which have an overall inhibitory effect on the cell. ${ }^{27}$

The management of analgesia by opioid administration occurs via the ability to bind to $\mu$-opioid receptors in the central system. However, gastrointestinal tract side effects become problematic because of the lack of selectivity of the opioids centrally; this leads to agonism of $\mu$-opioid receptors in the periphery, including those in the gastrointestinal tract. Within the gastrointestinal tract, there is an abundance of $\mu$-opioid receptors in the submucosa ${ }^{28}$ and in the ileal mucosa, where they affect ion transport and balance. ${ }^{29}$ The proximal colon and the stomach have the highest concentrated population of $\kappa$-opioid and $\mu$-opioid receptors. ${ }^{30}$ As a consequence, patients undergoing opioid therapy will often experience a disruption in the propulsive motility of the gastrointestinal tract along with a disturbance of the migrating motor complex, a lengthening of the duration of gastrointestinal transit time, and a decrease in secretion of gastric fluids and intestinal ions. ${ }^{31}$

\section{Treatment options for PDNP}

First and foremost, preventative measures are crucial to decrease the risks of the complications that accompany uncontrolled diabetes mellitus. Strict restriction of glycemic intake has been shown to help in the prevention of long-term complications. Other risk factors that should be addressed include obesity, smoking, heavy alcohol use, hyperlipidemia, hypertension, and vitamin B6 and B12 deficiency. ${ }^{12}$

At the time of this writing, the only antidepressants approved for the treatment of PDNP by the US Food and Drug Administration (FDA) are duloxetine and pregabalin, the latter of which is also the only anticonvulsant approved for PDNP by that agency. ${ }^{32}$ However, other medications are used as well for this purpose. ${ }^{32}$ Pure $\mu$-opioid agonists have shown an efficacy equivalent to that of pregabalin and gabapentin, ${ }^{17,33-38}$ but they fall short as optimal treatment options because of inadequate tolerability and are therefore usually not considered as front-line analgesic options for neuropathic pain. ${ }^{18,39}$ Because PDNP has a multifaceted pathophysiology, it often requires a multimodal approach to treatment. ${ }^{34}$ Consequently, an analgesic that targets pain through a combination of mechanisms may potentially provide the most effective treatment for PDNP.$^{40}$ Historically, a wide variety of medications have been used to target PDNP including tricyclic antidepressants, serotonin-norepinephrine reuptake inhibitors, antiepileptics, and opiates. Most recently, tapentadol has been studied extensively in the management of PDNP and has demonstrated not only clinical efficacy but also a better side effect profile. ${ }^{12}$

\section{Overview of the pharmacology, mode of action, pharmacokinetics of tapentadol extended release Overview}

Tapentadol is an opioid analgesic that was developed relatively recently by Gruenthal, a pharmaceutical company in 
Aachen, Germany, alongside Johnson \& Johnson Pharmaceutical Research and Development. ${ }^{41}$ Tapentadol is available in solution form or as an immediate-release (IR) tablet to target moderate to severe acute pain. It was approved by the FDA in 2009. ${ }^{42}$ An extended-release (ER) film coated tablet for oral administration was approved by the FDA in 2011 for the relief of moderate to severe chronic pain and later to address diabetic neuropathy, which necessitates continuous administration of the drug for a prolonged period. Tapentadol is graded as a US Drug Enforcement Administration Schedule II medication. ${ }^{43}$ It has also been approved in Europe to target severe chronic pain in adults who can only receive sufficient analgesia through opioids. ${ }^{44}$ As of 2010, tapentadol IR and tapentadol ER were approved in 26 European countries. ${ }^{45}$

Tapentadol has two demonstrated modes of action: it is a weak $\mu$-opioid receptor agonist and it is a norepinephrine reuptake inhibitor. It represents a new class of centrally acting opioid analgesic, the $\mu$-opioid receptor agonist-norepinephrine reuptake inhibitor. ${ }^{46,47}$ Stimulation of $\mu$-opioid receptors results in a diminution of relayed spinal pain and activity at the supraspinal levels via the descending pathway; this effect additionally reduces conduction of sensory signals. Furthermore, tapentadol's inhibitory effect on the reuptake of norepinephrine augments the inhibition of pain within the descending pathway, most possibly through $\alpha 2$ adrenoreceptors. $^{5}$

Because of tapentadol's weak affinity for the $\mu$-opioid receptor, there is a reduction in the common side effects that are associated with opioid use. Furthermore, its norepinephrine reuptake inhibiting ability lends to its efficacy as an analgesic drug. ${ }^{48}$ This has also improved the efficacy of therapeutic opioid use in the treatment of neuropathic pain. ${ }^{49}$ The dual action lends this drug the potential to diminish pain as a single drug functioning as a multimodal opioid - this could lessen the risk of any undesired drug-drug interactions. ${ }^{50}$

The manufacturer recommends an initial dose of $50 \mathrm{mg}$, $75 \mathrm{mg}$, or $100 \mathrm{mg}$ every 4-6 hours on the basis of pain intensity. However, a second dose can be given 1 hour after the initial dose if it was found to be insufficient. The maximum daily dose is $600 \mathrm{mg}$.

Given its relatively weaker $\mu$-opioid receptor affinity, tapentadol is believed to have less dependence and abuse potential than more traditional opioids. One rat model study did demonstrate the development of dependence to the anti-allodynic effects of tapentadol. ${ }^{47}$ In one comparative study, when the Clinical Opiate Withdrawal Scale was used, subjects taking tapentadol were found to be less likely to have withdrawal symptoms than subjects taking oxycodone (17\% versus $29 \%$ ); however, no differences were found between the two medications when the Subjective Opiate Withdrawal Scale was used. ${ }^{51}$ A recent retrospective cohort study has demonstrated the risk of receiving an abuse diagnosis with tapentadol to be lower than the risk with oxycodone..$^{52}$

\section{Chemical structure}

Tapentadol is made up of two chiral carbon atoms, and thereby exists as four stereoisomers. It is a pure enantiomer; analgesic activity is provided only by the $\mathrm{S}, \mathrm{S}$ (left handed configuration) and R, R (right handed configuration) isomers - the diastereoisomers are not active forms. ${ }^{53}$ The dimethylaminomethyl component of tapentadol is responsible for its activity with the $\mu$-opioid receptor. ${ }^{54}$ When demethylated, the resulting derivatives of the compound, $\mathrm{CH}_{2} \mathrm{NH}_{2}$ and $\mathrm{CH}_{2}-\mathrm{NH}\left(\mathrm{CH}_{3}\right)$, are devoid of any substantial analgesic activity. ${ }^{55,56}$

\section{Tapentadol ER}

Tapentadol ER is supplied in tablets of varying doses of tapentadol hydrochloride at $58.24 \mathrm{mg}, 116.48 \mathrm{mg}, 174.72 \mathrm{mg}$, $232.96 \mathrm{mg}$, and $291.20 \mathrm{mg}$. These increments respectively correspond to $50 \mathrm{mg}, 100 \mathrm{mg}, 150 \mathrm{mg}, 200 \mathrm{mg}$, and $250 \mathrm{mg}$ of free-base tapentadol. Aside from the active ingredient, tapentadol $\mathrm{HCl}$, the tablets contain the following inactive ingredients: polyethylene oxide, polyethylene glycol, hypromellose, and alpha-tocopherol. The film coating of the tablets contains polyvinyl alcohol, polyethylene glycol, talc, titanium dioxide, and yellow iron oxide for $150 \mathrm{mg}$ tablets, and Food, Drug, and Cosmetics (FD\&C) Blue \#2 for the $100 \mathrm{mg}$, $150 \mathrm{mg}, 200 \mathrm{mg}$, and $250 \mathrm{mg}$ tablets. The printing ink is made up of shellac glaze, propylene glycol, and titanium dioxide for the $250 \mathrm{mg}$ strength, and black iron oxide for the $50 \mathrm{mg}$, $100 \mathrm{mg}, 150 \mathrm{mg}$, and $200 \mathrm{mg}$ strengths. ${ }^{42}$

Tapentadol IR is available in three doses $(50 \mathrm{mg}, 75 \mathrm{mg}$, and $100 \mathrm{mg}$ ) given every 4-6 hours, whereas tapentadol ER is given twice a day. The formulations for IR are bioequivalent to ER and thereby allow for easy $1: 1$ conversions of doses. ${ }^{57}$ The maximum daily dose of tapentadol ER is designated as $500 \mathrm{mg}$ for every 24 hours, whereas that of IR is $600 \mathrm{mg}$ for every 24 hours aside from the first day of therapy, on which a maximum dose of $700 \mathrm{mg}$ is permitted to deliver a loading analgesic dose. ${ }^{58}$

\section{Metabolism}

The drug reaches maximum serum concentrations between 3 and 6 hours after administration. It has been observed to maintain concentrations at a steady-state following the third dose, which is given 24 hours following the first two 
administrations of the daily multiple doses. Furthermore, the most marginal levels of accumulation were observed with $250 \mathrm{mg}$ doses given every 12 hours. $^{42}$

Tapentadol does not require metabolic activation and does not have any active metabolites. Approximately $97 \%$ of the substance is metabolized in humans, predominantly through the Phase 2 pathways - only a small share of the compound goes through Phase 1 oxidative pathways. The favored metabolic pathway of tapentadol yields glucoronides after conjugation with glucoronic acid. After oral administration, tapentadol undergoes sulfation and direct glucuronidation ( $15 \%$ sulfate, $55 \%$ O-glucuronide); roughly $70 \%$ of the final products are excreted in urine as conjugated end-products. ${ }^{42}$ Tapentadol undergoes glucuronidation via UGT2B7 and UGT1A9. ${ }^{59}$ About $13 \%$ of tapentadol is also metabolized to N-desmethyl tapentadol via CYP2C19 and CYP2C9, and approximately $2 \%$ to hydroxyl tapentadol via CYP2D6, both of which are further conjugated. The main metabolite of tapentadol, tapentadol-O-glucoronide, does not activate synaptosomal reuptake systems, opioid receptors, or any other binding sites. ${ }^{47}$ Approximately 3\% of the compound is excreted in urine as an unmetabolized, unaltered drug. ${ }^{42}$

Considering what is presently understood about the pathways of metabolism of this drug, it is thought to be unlikely to have its clinical applications complicated by drug-drug interactions. Studies have shown that this drug does not induce production of CYP1A2, CYP2A6, CYP2C9, CYP2C19, CYP2E1, or CYP3A4 at any concentration. ${ }^{60}$ Moreover, no autoinduction in fresh human hepatocytes was observed, suggesting that tapentadol is not likely to affect the metabolism of concurrently managed drugs that are metabolized via the CYP pathway. ${ }^{60}$ Furthermore, because the plasma protein binding is low, it is unlikely that tapentadol would cause clinically significant displacement of concomitantly administered drugs. These two factors together make tapentadol highly unlikely to exhibit clinically significant drug-drug interactions with drugs affected by CYP or plasma proteinbinding activity at usual plasma concentrations. ${ }^{60}$

Both tapentadol and its metabolites are eliminated almost entirely via the kidneys (99\%). Following oral administration, the drug has a half-life averaging roughly 5 hours. The total clearance of the drug is $1,603 \pm 227 \mathrm{~mL} / \mathrm{min} .{ }^{42}$

\section{Pharmacokinetics}

Tapentadol has been calculated to have a $\mathrm{K}_{\mathrm{i}}$ of $0.5 \mu \mathrm{M}$ in rat synaptosomal norepinephrine reuptake assays and $0.1 \mu \mathrm{M}$ in $\mu$-opioid receptor binding assays. ${ }^{47}$ These characteristics indicate that tapentadol's $\mu$-opioid receptor has an affinity that is 50 times lower than that of morphine. Levels of affinity at other types of opioid receptors, including that of the ORL1 receptor, the $\delta$-opioid receptor, and the $\kappa$-opioid receptor, are at least one order of magnitude less than at the $\mu$-opioid receptor. ${ }^{47}$

\section{Efficacy studies that include comparative studies}

Tapentadol has demonstrated clinical efficacy in improving pain scores in patients with a wide variety of painful disease states, such as chronic back pain, cancer pain, and PDNP. Because of tapentadol's distinct pharmacology and mode of action, studies have been able to demonstrate not only improved gastrointestinal side effect profiles, but also better adherence and less tolerance potential. Much of the most recently available data suggests that tapentadol may have particular clinical utility in the management of PDNP.

Tapentadol's modest affinity for the $\mu$-opioid receptor and the drug's opioid-sparing effects, which are caused by its norepinephrine reuptake inhibitory characteristic, imply that the drug should generate fewer side effects than morphine and other conventional $\mu$-opioid receptor agonists at equianalgesic dosages. In a study that compared the side effects of morphine with those of tapentadol in ferrets, it was found that tapentadol caused much less frequent vomiting and shorter durations of vomiting. In addition, the dosage that was needed to elicit these responses was 100 times smaller for morphine than for tapentadol. These data demonstrate that tapentadol is a weaker contributor to gastrointestinal side effects than morphine. ${ }^{46}$

Studies have observed that tapentadol ER is an effective analgesic to target moderate to severe chronic osteoarthritisrelated knee pain. Treatment using tapentadol ER yielded a lower occurrence of gastrointestinal side effect disorders in a comparative study against oxycodone controlled-release (CR), which is an opioid agonist commonly prescribed for this population. ${ }^{61} \mathrm{~A}$ follow-up study demonstrated significantly higher at-work productivity in patients with osteoarthritic chronic moderate to severe pain who were taking tapentadol ER than in subjects who were taking oxycodone CR or a placebo. ${ }^{62}$ Similarly, another study of patients taking a placebo, tapentadol ER, or oxycodone CR for either lower back pain or osteoarthritis found that those on tapentadol ER had greater work productivity than those on oxycodone CR. The measurements of increased productivity included fewer absences and a decrease in overall loss of work productivity. ${ }^{45}$

Clinical trials in patients suffering from chronic lower back pain similarly found that tapentadol offered fewer 
occurrences of adverse side effects than did oxycodone within equivalent doses. ${ }^{63}$ A recent study demonstrated significantly greater improvements in pain intensity measures for patients with severe chronic osteoarthritis knee pain who had previously responded to World Health Organization Step III therapy and who were later treated with tapentadol ER (50-250 mg twice daily) than patients who were treated with step III opioids. Interestingly, the improvements observed in pain intensity scores in the tapentadol ER group were accompanied with improvements in anxiety and depression, function, quality-of-life, and health status. ${ }^{64}$

Tapentadol's synergistic mechanism of activity as both a $\mu$-opioid receptor agonist and a norepinephrine reuptake inhibitor has also other demonstrated advantages. The time required for patients to develop tolerance is longer with tapentadol than morphine. In fact, it was shown that tolerance to morphine developed 2.5 times more rapidly than to tapentadol. ${ }^{47}$ Furthermore, tapentadol's characteristic dual mechanism leads to a smaller adverse event (AE) profile than that of other opioids. ${ }^{58}$

Polypharmacy is an area of concern particularly in elderly patients who commonly take more than ten medications. Particular sensitivity among the elderly population to AEs leads to poor compliance, greater risks of drug-drug interactions, and duplication of treatments. ${ }^{65}$ Thus, treatment options that offer combined multiple modes of action at low doses in a single medication confer significant opioid-sparing advantages to the elderly patient population in that these patients experience greater relief from pain while simultaneously experiencing fewer AEs. ${ }^{66}$ Indeed, in practice, the clinical grouping of a monoamine reuptake inhibitor with an opioid agonist seems to minimize AEs and improve pain management. ${ }^{47,67-69}$ The first pharmacological drug that was available to provide these dual actions within a single medication was tramadol. Tapentadol was developed to overcome the impediments to tramadol's effectiveness. ${ }^{47}$

In addition to improvement of opioid-induced gastrointestinal side effects, and perhaps largely because of it, administration of tapentadol ER seems to also lower the incidence of patient-initiated study discontinuation due to treatment-emergent AEs (TEAEs) more than oxycodone CR does. In one study, for example, 981 patients were randomized to receive tapentadol ER, oxycodone CR, or a placebo over 15 weeks, and the tapentadol group demonstrated a lower incidence of TEAEs, including constipation, nausea, and vomiting, than did the oxycodone group. As a result, tapentadol ER may offer a more tolerable and steady treatment to managing chronic pain. ${ }^{70}$ Similarly, a pooled analysis of three Phase III studies involving a total of 2,968 study participants demonstrated that tapentadol ER (100-250 mg twice daily) had a similar efficacy to oxycodone $\mathrm{HCl} \mathrm{CR}$ (20-50 mg twice daily) for the management of chronic osteoarthritis knee and low back pain and that tapentadol ER had a superior gastrointestinal tolerability profile and fewer treatment discontinuations. ${ }^{71}$

The application of tapentadol ER to manage cancer pain was initially studied by Mercadante et al. ${ }^{72-75}$ Only $7 \%$ of the patients in this cohort discontinued usage of tapentadol or showed poor compliance with its treatment; this finding suggests that tapentadol is a well-tolerated drug in cancer patients. Furthermore, the rate of discontinuation of tapentadol was less than that of other therapeutic opioids that had been formerly considered, including transdermal buprenorphine (15\%), transdermal fentanyl (14\%), and oral morphine $(13 \%) .{ }^{72-75}$ In a recent double-blind randomized controlled study of 236 patients with chronic malignant tumor-related cancer pain, tapentadol ER was compared to oxycodone CR in terms of analgesic efficacy and TEAEs. The study demonstrated that tapentadol ER provides analgesic efficacy that is not inferior to that of oxycodone in this particular patient population and, at the same time, results in a better gastrointestinal tolerability profile. ${ }^{76}$ Another recent double-blind Phase III randomized placebo-controlled study that involved 496 patients with moderate to severe chronic malignant tumor-related pain compared tapentadol ER (100-250 mg administered twice a day) to morphine sulfate CR (40-100 mg administered twice a day). The study demonstrated tapentadol to be more effective than a placebo and that while tapentadol provides efficacy comparable to that of morphine, tapentadol has superior gastrointestinal tolerability. ${ }^{77}$

Another group of patients that appears to benefit from tapentadol ER is the group with chronic back pain with a neuropathic component. Baron et al studied the effects of $300 \mathrm{mg}$ per day of tapentadol ER in patients with severe chronic low back pain with a neuropathic component; the study demonstrated significant improvements in pain intensity, neuropathic pain-related symptoms, and quality of life. ${ }^{78}$ The same research group compared the effectiveness and tolerability of tapentadol ER monotherapy and tapentadol $\mathrm{ER} /$ pregabalin combination therapy in patients with chronic low back pain with a neuropathic component. The doubleblind randomized trial demonstrated improved neuropathic pain scores and quality-of-life variables along with improved tolerability in both treatment groups. Interestingly, tapentadol alone was associated with a lower incidence of dizziness and somnolence than the combination of tapentadol ER and 
pregabalin (16.9\% versus $27.0 \%){ }^{78}$ Tapentadol, therefore, appears to have particular clinical utility in treating chronic back pain with a neuropathic component.

Tapentadol ER has been associated with a more favorable side effect profile than traditional opioid medications, especially with regard to nausea and other gastrointestinal symptoms. Mercadante et al observed that patients who shifted therapy to morphine from tapentadol presented with more unfavorable side effects as measured by an increase in the distress score (predominantly attributed to vomiting and nausea) and the usage of antiemetic drugs. ${ }^{73}$ On the other hand, subjects who converted from taking morphine to tapentadol showed no differences in the distress scores. ${ }^{73}$ In a recent randomized trial in patients with chronic malignant tumor-related cancer pain on around-the-clock strong opioid therapy, the patients were randomized $(1: 1)$ to receive twicedaily treatment with tapentadol ER or morphine sustained release. In the tapentadol group, 84\% maintained pain control after 1 week, whereas $98 \%$ did so in the morphine group. However, the tapentadol group exhibited a lower incidence of gastrointestinal side effects. ${ }^{79}$

\section{Tapentadol and PDNP}

Numerous experiments have also been conducted that exclusively analyze the efficacy of tapentadol in the management of PDNP. A recent study has demonstrated that tapentadol potentiates descending pain inhibition in chronic pain patients with diabetic polyneuropathy. The study employed two experimental paradigms of endogenous pain modulation: 1) conditioned pain modulation, an experimental measure of endogenous pain inhibition that gates incoming pain signals as a consequence of a preceding painful stimulus; and 2) offset analgesia, a test in which a large amount of analgesia becomes apparent upon a slight decrease in noxious heat stimulation. Twenty-four patients with diabetic polyneuropathy were randomized to receive daily treatment with tapentadol ER or placebo for 4 weeks. At the conclusion of the study period, tapentadol ER demonstrated significant pain relief that coincided with enhanced conditioned pain modulation responses. No effect of tapentadol was observed in the responses to offset analgesia. Additionally, relief of diabetic neuropathic pain was also greater in patients treated with tapentadol than in those treated with placebo $(P=0.028)$. Thus, the specific effects of tapentadol on diabetic neuropathic pain may be mediated by the potentiation of descending inhibitory pain pathways. ${ }^{80}$

In a study that was conducted by Schwartz et al (2011) on diabetic patients who had suffered from diabetic neuropathic pain for at least 6 months, tapentadol ER 100-250 mg administered twice a day was found to be well tolerated and also to improve experienced pain significantly more than a placebo. ${ }^{40}$ From the beginning of the double-blind treatment to week 12 , the average increase in mean pain intensity in the placebo group was 1.4. However, there was no change in the calculated mean pain intensity within this range in the group treated with tapentadol ER (0.0). There were no substantial differences between female and male patients for the average changes in mean pain intensity scores in the double-blind treatment from the beginning through week 12 of treatment in the tapentadol ER group. There were also no substantial differences in changes in the mean pain intensities between opioid-experienced and opioid-naive subjects within this group. Of the patients in this group, $65 \%$ reported an improvement of at least $30 \%$ in pain intensity, and $34.9 \%$ experienced an improvement of at least $50 \%$ in pain intensity.

Similar results were demonstrated in a recent randomized placebo-controlled Phase III study of the efficacy and tolerability of tapentadol ER in the management of chronic neuropathic pain associated with PDNP. This second study was similar in design to the one conducted by Schwartz et al in 2011, except this study used a new formulation of tapentadol ER that is approved in the US for the treatment of chronic pain. This new formulation of tapentadol has a high mechanical strength conferred by the use of a polyethylene oxide matrix and melt extrusion manufacturing process, and this formulation is less susceptible to breakage, splitting, crushing, or chewing than the conventional hypromellosebased formulation used in other Phase III tapentadol ER studies. ${ }^{81}$ In the study, adults with moderate to severe diabetic neuropathic pain were titrated to tapentadol ER 100-250 mg twice a day during a 3-week open-label period; patients with $\geq 1$-point reduction in pain intensity at end of titration (number of patients $[n]=318$ ) received either a placebo or tapentadol ER (optimal dose from titration) for 12 weeks. After the 12-week period, the mean change in pain intensity from the start of double-blind treatment to week 12 was 1.30 in the placebo group and 0.28 in the tapentadol ER group. TEAEs ( $\geq 10 \%$ ) in the tapentadol ER group during the double-blind maintenance phase were nausea $(21.1 \%)$ and vomiting (12.7\%); these findings demonstrate a similar tolerability profile to profiles from previous studies. ${ }^{81}$

Tapentadol ER has demonstrated acceptable tolerability and clinically efficacy in reduction of pain intensity in two recent clinical studies of patients with diabetic neuropathic pain. Diabetic neuropathic pain, which affects up to $25 \%$ of 
all patients with diabetes, has proven to be a source of often debilitating pain in this population of patients. Tapentadol, a unique, centrally acting analgesic with both opioid receptor agonism and norepinephrine reuptake inhibition, appears to be well tolerated and efficacious in treating pain from PDNP.

\section{Safety and tolerability issues}

The foremost concern regarding tapentadol in terms of safety is its effect on respiratory depression. Tapentadol causes depression of the respiratory system via its effect on the respiratory centers in the brainstem, and therefore the drug should not be administered to patients with severe respiratory illnesses. Furthermore, elderly and compromised patients with an underlying respiratory disease should refrain from taking this drug or take it in the lowest sufficient dose of effectiveness. The tablets must not be broken, smashed, or fragmented because of potentially fatal effects that can follow rapid absorption. ${ }^{82}$

Several studies have assessed the tolerability of tapentadol and compared it to that of conventional opioid therapy. In a large, randomized open-label Phase III study of patients with chronic knee or hip osteoarthritis pain or low back pain, patients were randomized to receive twice-daily doses of tapentadol ER (100-250 mg) or oxycodone HCl CR (20-50 mg) for up to 1 year. The incidences of several types of TAEAs were lower for the tapentadol group; these include constipation $(22.6 \%$ versus $38.6 \%$ ), nausea (18.1\% versus $33.2 \%$ ), vomiting ( $7 \%$ versus $13.5 \%)$, and dizziness ( $14.8 \%$ versus $19.3 \%$ ). Furthermore, TEAEs led to discontinuation less frequently in the tapentadol group than in the oxycodone group (22.1\% versus $36.8 \%)$. In a recent analysis of three randomized controlled trials, patients with hypertension who had been treated with tapentadol ER were found to have no clinically meaningful changes in blood pressure or heart rate. ${ }^{83}$

In a recent analysis of pooled data from four randomized controlled Phase III studies in over 4,000 patients with chronic osteoarthritis hip pain, knee pain, or low back pain, tapentadol ER (100-250 mg twice a day) demonstrated better gastrointestinal tolerability than did oxycodone CR. The analysis considered the overall incidence of gastrointestinal TEAEs, the incidence of moderate or severe gastrointestinal TEAEs, and the incidence of gastrointestinal TEAEs leading to discontinuation. ${ }^{84}$ Therefore, while tapentadol has some of the same serious AEs as standard opioids do, such as respiratory depression, tapentadol is associated with fewer gastrointestinal symptoms and, for this reason, appears to be better tolerated than conventional opioid therapies. ${ }^{85}$
To date, there have been no adequately controlled studies of tapentadol used by women during pregnancy. A meta-analysis found no clear evidence of fetal or neonatal harm, but a possible increased rate of fetal loss has been reported when the drug is taken early in the pregnancy. ${ }^{86}$ The same article states that it appears unlikely that maternal use during breastfeeding will cause harm to healthy-term infants. Without well controlled studies, the drug should be used during pregnancy only if the potential benefit justifies the potential risk to the fetus. ${ }^{87}$

\section{Compliance and cost Compliance}

As important as the efficacy of the drug in question is its rate of compliance in the patient population, for offering a mechanistically effective treatment is unserviceable if the subjects do not consistently comply with the recommended administration or dosages. In a study conducted by Coluzzi and Ruggeri (2014) on patients experiencing musculoskeletal pain, researchers observed that the expected risk ratio (RR) of withdrawal from treatment because of AEs was significantly lower for tapentadol ER (RR 0.526; 95\% CI 0.4656-0.607) than for $\mathrm{CR}$ oxycodone and for naloxone/oxycodone ER (RR 0.758; 95\% CI 0.427-1.343). ${ }^{88}$

When administered to diabetic patients suffering from PDNP, the compliance rates for tapentadol ER were similar to those observed in previous studies. ${ }^{89,90}$ Roughly $30 \%$ of the subjects terminated tapentadol ER treatment during the double-blind or open-label titration period, and about 30\% of the subjects terminated treatment with placebo during the period of double-blind treatment. Moreover, the occurrence of AEs, including nausea and vomiting, that lead to the termination of treatment was comparable in this study (vomiting, 2.7\%; nausea, 7.7\%) to those in studies conducted on patients with chronic lower back pain ${ }^{70}$ and to those in studies on patients with osteoarthritic pain. ${ }^{85}$

\section{Cost}

The expenses that accumulate in the treatment of neuropathic pain are considerable. This presents dually as the loss caused by the decrease in work productivity and as the direct and indirect medical expenses. For instance, in a survey of patients in Europe with neuropathic pain, within the month preceding the survey, $19 \%$ of respondents had been to a physician three or more times, and $49 \%$ had visited a pain specialist. Patients suffering from neuropathic pain often were also treated for anxiety, sleep disorders, and depression. ${ }^{91-94}$

An aforementioned study calculated the projected average expense savings per year for each subject and found that 
the mean savings were $\$ 1,400$ for oxycodone $C R, \$ 1,510$ for placebo, and $\$ 1,960$ for tapentadol ER. ${ }^{62}$ Based on an annual salary figure of $\$ 100,000$, the estimated net savings were $\$ 450$ per subject with tapentadol ER and $\$ 560$ per subject with oxycodone CR treatment. ${ }^{62}$ In effect, tapentadol can potentially offer patients a low-cost alternative to more commonplace opioids such as oxycodone.

In Spain, a similar study was conducted to compare the cost-effectiveness of tapentadol with transdermal fentanyl and morphine. Calculations confirmed the economic advantages of tapentadol over other available analgesic drugs; there is an estimated incremental cost-effectiveness ratio of $€ 2,656$ for morphine and $€ 2,069$ for transdermal fentanyl. Additionally, the likelihood of tapentadol being more cost-effective than these other drugs at the "willingness-to-pay threshold" between $€ 20,000$ and $€ 30,000$ per quality-adjusted life-year gained based on the National Spanish Health System surpassed 90\%. ${ }^{95}$

A recent meta-analysis employed a Markov model to compare the cost effectiveness of tapentadol ER and oxycodone/naloxone ER for the treatment of musculoskeletal pain by indirect comparison with $\mathrm{CR}$ oxycodone. In this study both analgesics were shown to be more cost effective than CR oxycodone. Furthermore, in $65 \%$ of cases, tapentadol ER was not only less costly but also produced a gain in quality-adjusted life-years. The authors hypothesize that the superior cost effectiveness of tapentadol ER was likely related to the price, the lower incidence of AEs, and the lower discontinuation rate related to the AEs. ${ }^{88}$

\section{Conclusion}

The growing numbers in preclinical and clinical data suggest tapentadol's efficacy in a wide array of patient populations and across numerous etiologies of traditionally difficult to manage pain. The outcomes of numerous studies portray the growing potential of tapentadol as a treatment modality that offers greater efficacy and tolerability than classical opioids. Several recent clinical trials have demonstrated improved outcomes in pain indices and quality of life measures of patients treated with tapentadol. Interestingly, the novel analgesic appears to be more tolerable in patients who have previously failed treatment with more conventional opioid therapy. Chronic pain due to PDNP appears to be particularly responsive to tapentadol; this finding is promising given the increased societal burden and recalcitrant nature of this pain. Emerging data also suggest that tapentadol is more cost-effective and has better tolerability and clinical outcomes than more conventional opioid-based therapies. There remains a need to further investigate the effectiveness of tapentadol in comparison with currently available analgesic drugs so that in the future, treatments can be tailored to the individual patient.

\section{Disclosure}

The authors report no conflicts of interest in this work.

\section{References}

1. Merskey H, Bogduk N, editors. Part III: pain terms, a current list with definitions and notes on usage. In: Classification of chronic pain, IASP task force on taxonomy, 2nd ed. Seattle: IASP Press; 1994:209-214.

2. Hammersla M, Kapustin JF. Peripheral Neuropathy: Evidence-based treatment of a complex disorder. Nurse Pract. 2012;37(5):32-39; quiz $39-40$.

3. Wiggin TD, Sullivan KA, Pop-Busui R, Amato A, Sima AA, Feldman EL. Elevated triglycerides correlate with progression of diabetic neuropathy. Diabetes. 2009;58(7):1634-1640.

4. Zhuo M, Wu G, Wu LJ. Neuronal and microglial mechanisms of neuropathic pain. Mol Brain. 2011;4(31):1.

5. Kress HG. Tapentadol and its two mechanisms of action: is there a new pharmacological class of centrally-acting analgesics on the horizon? European J Pain. 2010;14(8):781-783.

6. Cohen SP, Mao J. Neuropathic pain: mechanisms and their clinical implications. BMJ. 2014;348:f7656.

7. Wall P, Devor M. Sensory afferent impulses originate from dorsal root ganglia as well as from the periphery in normal and nerve injured rats. Pain. 1983;17(4):321-339.

8. Bannister K, Bee LA, Dickenson AH. Preclinical and early clinical investigations related to monoaminergic pain modulation. Neurotherapeutics. 2009;6(4):703-712.

9. Azhary H, Farooq MU, Bhanushali M, Majid A, Kassab MY. Peripheral neuropathy: differential diagnosis and management. Am Fam Phys. 2010;81(7):887-892.

10. Neville A, Peleg R, Singer Y, Sherf M, Shvartzman P. Chronic pain: a population-based study. Isr Med Assoc J. 2008;10(10):676-680.

11. Liberman O, Peleg R, Shvartzman P. Chronic pain in type 2 diabetic patients: A cross-sectional study in primary care setting. Eur J Gen Pract. 2014;20(4):260-267.

12. Iyer S, Tanenberg RJ. Pharmacologic management of diabetic peripheral neuropathic pain. Expert Opin Pharmacother. 2013;14(13): 1765-1775.

13. Henningsen $P$, Zipfel S, Herzog W. Management of functional somatic syndromes. The Lancet. 2007;369(9565):946-955.

14. Bansal V, Kalita J, Misra U. Diabetic neuropathy. Postgrad Med J. 2006;82(964):95-100

15. Hjalte F, Berggren A-C, Bergendahl H, Hjortsberg C. The direct and indirect costs of opioid-induced constipation. J Pain Symptom Manage. 2010;40(5):696-703.

16. Avouac J, Gossec L, Dougados M. Efficacy and safety of opioids for osteoarthritis: a meta-analysis of randomized controlled trials. Osteoarthritis Cartilage. 2007;15(8):957-965.

17. Eisenberg E, McNicol ED, Carr DB. Efficacy and safety of opioid agonists in the treatment of neuropathic pain of nonmalignant origin systematic review and meta-analysis of randomized controlled trials. JAMA. 2005;293(24):3043-3052.

18. Dworkin RH, O'Connor AB, Backonja M, et al. Pharmacologic management of neuropathic pain: evidence-based recommendations. Pain 2007;132(3):237-251.

19. Kalso E, Edwards JE, Moore RA, McQuay HJ. Opioids in chronic noncancer pain: systematic review of efficacy and safety. Pain. 2004;112(3) 372-380.

20. Gregorian Jr RS, Gasik A, Kwong WJ, Voeller S, Kavanagh S. Importance of side effects in opioid treatment: a trade-off analysis with patients and physicians. J Pain. 2010;11(11):1095-1108. 
21. Nicholson B. Responsible prescribing of opioids for the management of chronic pain. Drugs. 2003;63(1):17-32.

22. Benyamin R, Trescot AM, MD, Datta S, et al. Opioid complications and side effects. Pain Physician. 2008;11(2 Suppl):S105-S120.

23. Bell TJ, Panchal SJ, Miaskowski C, Bolge SC, Milanova T, Williamson R. The prevalence, severity, and impact of opioid-induced bowel dysfunction: results of a US and European patient survey (PROBE 1). Pain Med. 2009;10(1):35-42.

24. Panchal SJ, Müller-Schwefe P, Wurzelmann JI. Opioid-induced bowel dysfunction: prevalence, pathophysiology and burden. Int J Clin Pract. 2007;61(7):1181-1187.

25. Pappagallo M. Incidence, prevalence, and management of opioid bowel dysfunction. Am J Surg. 2001;182(5A Suppl):11S-18S.

26. Thorpe DM. Management of opioid-induced constipation. Curr Pain Headache Rep. 2001;5(3):237-240.

27. Rang HP, Dale MM, Ritter JK, Moore PK. Analgesic drugs. Pharmacology. 1999;4:587.

28. Bagnol D, Mansour A, Akil H, Watson SJ. Cellular localization and distribution of the cloned mu and kappa opioid receptors in rat gastrointestinal tract. Neuroscience. 1997;81(2):579-591.

29. McKay JS, Linaker BD, Turnberg LA. Influence of opiates on ion transport across rabbit ileal mucosa. Gastroenterology. 1981;80(2): 279-284.

30. Fickel J, Bagnol D, Watson SJ, Akil H. Opioid receptor expression in the rat gastrointestinal tract: a quantitative study with comparison to the brain. Brain Res Mol Brain Res. 1997;46(1-2):1-8.

31. Holzer P. Opioids and opioid receptors in the enteric nervous system: from a problem in opioid analgesia to a possible new prokinetic therapy in humans. Neurosci Lett. 2004;361(1-3):192-195.

32. Veves A, Backonja M, Malik RA. Painful diabetic neuropathy: epidemiology, natural history, early diagnosis, and treatment options. Pain Med. 2008;9(6):660-674.

33. Finnerup NB, Otto M, McQuay HJ, Jensen TS, Sindrup SH. Algorithm for neuropathic pain treatment: an evidence based proposal. Pain. 2005; 118(3):289-305.

34. Argoff CE, Backonja MM, Belgrade MJ, et al. Consensus guidelines: treatment planning and options. Mayo Clinic Proceedings. 2006; 81(4):S12-S25.

35. Gimbel JS, Richards P, Portenoy RK. Controlled-release oxycodone for pain in diabetic neuropathy: a randomized controlled trial. Neurology. 2003;60(6):927-934.

36. Watson CP, Moulin D, Watt-Watson J, Gordon A, Eisenhoffer J. Controlled-release oxycodone relieves neuropathic pain: a randomized controlled trial in painful diabetic neuropathy. Pain. 2003;105(1-2): 71-78.

37. Watson CP, Babul N. Efficacy of oxycodone in neuronathic pain: a randomized trial in postherpetic neuralgia. Neurology. 1998;50(6): $1837-1841$.

38. Harati Y, Gooch C, Swenson M, et al. Double-blind randomized trial of tramadol for the treatment of the pain of diabetic neuropathy. Neurology. 1998;50(6):1842-1846.

39. Attal N, Cruccu G, Haanpää M, et al. EFNS guidelines on pharmacological treatment of neuropathic pain. Eur J Neurol. 2006;13(11):1153-1169.

40. Schwartz S, Etropolski M, Shapiro DY, et al. Safety and efficacy of tapentadol ER in patients with painful diabetic peripheral neuropathy: results of a randomized-withdrawal, placebo-controlled trial. Curr Med Res Opin. 2011;27(1):151-162.

41. Grünenthal GmbH. Grünenthal GmbH Presents Tapentadol, a Novel Centrally Acting Analgesic, at the 25th Annual Scientific Meeting of The American Pain Society [press release]. London, UK; PR Newswire; 2006. Available from: http://www.prnewswire.co.uk/news-releases/ grunenthal-gmbh-presents-tapentadol-a-novel-centrally-actinganalgesic-at-the-25th-annual-scientific-meeting-of-the-american-painsociety-153559155.html. Accessed October 22, 2014.

42. Nucynta (tapentadol) [package insert] Titusville, NJ: Janssen Pharmaceuticals; 2009.
43. US Food and Drug Administration. Medication Guide. Silver Spring, MD: US Food and Drug Administration; 2011. Available from: http:// www.accessdata.fda.gov/drugsatfda_docs/label/2011/200533s000lbl. pdf. Accessed October 10, 2014.

44. Palexia. Palexia SR (tapentadol) prolonged-release oral tablets: Summary of product characteristics. Buckinghamshire, UK: Grunenthal Ltd; 2011

45. Cepeda MS, Sutton A, Weinstein R, Kim M. Effect of tapentadol extended release on productivity: results from an analysis combining evidence from multiple sources. Clin J Pain. 2012;28(1):8-13.

46. Tzschentke T, De Vry J, Terlinden R, et al. Tapentadol hydrochloride. Drugs Future. 2006;31(12):1053-1061.

47. Tzschentke TM, Christoph T, Kögel B, et al. (-)-(1R, 2R)-3(3-Dimethylamino-1-ethyl-2-methyl-propyl)-phenol Hydrochloride (Tapentadol HCl): a Novel $\mu$-Opioid Receptor Agonist/Norepinephrine Reuptake Inhibitor with Broad-Spectrum Analgesic Properties. J Pharmacol Exp Ther. 2007;323(1):265-276.

48. Varrassi G, Marinangeli F, Piroli A, Coaccioli S, Paladini A. Strong analgesics: working towards an optimal balance between efficacy and side effects. Eur J Pain. 2010;14(4):340-342.

49. Wade WE, Spruill WJ. Tapentadol hydrochloride: a centrally acting oral analgesic. Clin Ther. 2009;31(12):2804-2818.

50. Hartrick C, Rozek RJ. Tapentadol in pain management: a $\mu$-opioid receptor agonist and noradrenaline reuptake inhibitor. CNS Drugs. 2011;25(5):359-370.

51. Hale M, Upmalis D, Okamoto A, Lange C, Rauschkolb C. Tolerability of tapentadol immediate release in patients with lower back pain or osteoarthritis of the hip or knee over 90 days: a randomized, doubleblind study. Curr Med Res Opin. 2009;25(5):1095-1104.

52. Cepeda MS, Fife D, Ma Q, Ryan PB. Comparison of the risks of opioid abuse or dependence between tapentadol and oxycodone: results from a cohort study. J Pain. 2013;14(10):1227-1241.

53. Raffa RB, Buschmann H, Christoph T, et al. Mechanistic and functional differentiation of tapentadol and tramadol. Expert Opin Pharmacother. 2012;13(10):1437-1449.

54. Casy AF, Parfitt RT. Opioid Analgesics. New York, NY: Plenum Press; 1986.

55. Maul C, Buschmann H, Sundermann B. Synthetic opioids. In: Buschmann H, Christoph T, Friderichs E, Maul C, Sunermann B, editors. Analgesics: From Chemistry and Pharmacology to Clinical Application. Weinheim: Wiley-VCH Verlag GmbH \& Co. KGaA; 2002:159-169.

56. Friderichs E, Buschmann H. Opioids: 3.4 Opioids with Clinical Relevance. In: Buschmann H, Christoph T, Friderichs E, Maul C, Sunermann B, editors. Analgesics: From Chemistry and Pharmacology to Clinical Application. Wiley-VCH Verlag GmbH \& Co. KGaA; 2002: 171-245.

57. Göhler K, Brett M, Smit JW, Rengelshausen J, Terlinden R. Comparative pharmacokinetics and bioavailability of tapentadol following oral administration of immediate-and prolonged-release formulations. Int J Clin Pharmacol Ther. 2013;51(4):338-348.

58. Atkinson TJ, Fudin J, Pandula A, Mirza M. Medication pain management in the elderly: unique and underutilized analgesic treatment options. Clin Ther. 2013;35(11):1669-1689.

59. Afilalo M, Stegmann JU, Upmalis D. Tapentadol immediate release: a new treatment option for acute pain management. J Pain Res. 2010;3:1-9.

60. Kneip C, Terlinden R, Beier H, Chen G. Investigations into the drugdrug interaction potential of tapentadol in human liver microsomes and fresh human hepatocytes. Drug Metab Lett. 2008;2(1):67-75.

61. Afilalo M, Etropolski MS, Kuperwasser B, et al. Efficacy and safety of tapentadol extended release compared with oxycodone controlled release for the management of moderate to severe chronic pain related to osteoarthritis of the knee: a randomized, double-blind, placeboand active-controlled phase III study. Clin Drug Invest. 2010;30(8): 489-505. 
62. Lerner D, Chang H, Rogers WH, et al. Imputing at-work productivity loss using results of a randomized controlled trial comparing tapentadol extended release and oxycodone controlled release for osteoarthritis pain. J Occup Environ Med. 2012;54(8):933-938.

63. Pergolizzi J, Alon E, Baron R, et al. Tapentadol in the management of chronic low back pain: a novel approach to a complex condition? J Pain Res. 2011;4:203-210.

64. Steigerwald I, Schenk M, Lahne U, Gebuhr P, Falke D, Hoggart B. Effectiveness and tolerability of tapentadol prolonged release compared with prior opioid therapy for the management of severe, chronic osteoarthritis pain. Clin Drug Invest. 2013;33(9):607-619.

65. Davis RG, Hepfinger CA, Sauer KA, Wilhardt MS. Retrospective evaluation of medication appropriateness and clinical pharmacist drug therapy recommendations for home-based primary care veterans. Am $J$ Geriatr Pharmacother. 2007;5(1):40-47.

66. Fudin J. Opioid pain management: balancing risks and benefits. Drug Topics. 2011;155(9):46-58.

67. Codd EE, Shank RP, Schupsky JJ, Raffa RB. Serotonin and norepinephrine uptake inhibiting activity of centrally acting analgesics: structural determinants and role in antinociception. J Pharmacol Exp Ther. 1995;274(3):1263-1270.

68. Malseed RT, Goldstein FJ. Enhancement of morphine analgesia by tricyclic antidepressants. Neuropharmacology. 1979;18(10):827-829.

69. Botney M, Fields HL. Amitriptyline potentiates morphine analgesia by a direct action on the central nervous system. Ann Neurol. 1983;13(2):160-164.

70. Buynak R, Shapiro DY, Okamoto A, et al. Efficacy and safety of tapentadol extended release for the management of chronic low back pain: results of a prospective, randomized, double-blind, placebo-and active-controlled Phase III study. Expert Opin Pharmacother. 2010;11(11):1787-1804.

71. Lange B, Kuperwasser B, Okamoto A, et al. Efficacy and safety of tapentadol prolonged release for chronic osteoarthritis pain and low back pain. Adv Ther. 2010;27(6):381-399

72. Mercadante S, Porzio G, Ferrera P, Aielli F, Adile C, Ficorella C. Low doses of transdermal fentanyl in opioid-naive patients with cancer pain. Curr Med Res Opin. 2010;26(12):2765-2768.

73. Mercadante S, Porzio G, Ferrera P, et al. Low doses of transdermal buprenorphine in opioid-naive patients with cancer pain: a 4-week, nonrandomized, open-label, uncontrolled observational study. Clin Ther. 2009;31(10):2134-2138.

74. Mercadante S, Porzio G, Ferrera P, et al. Low morphine doses in opioid-naive cancer patients with pain. J Pain Symptom Manage. 2006; 31(3):242-247.

75. Mercadante S, Porzio G, Ferrera P, et al. Tapentadol in cancer pain management: a prospective open-label study. Curr Med Res Opin. 2012;28(11):1775-1779.

76. Imanaka K, Tominaga Y, Etropolski M, et al. Efficacy and safety of oral tapentadol extended release in Japanese and Korean patients with moderate to severe, chronic malignant tumor-related pain. Curr Med Res Opin. 2013;29(10):1399-1409.

77. Kress HG, Koch E, Kosturski H. Tapentadol prolonged release for managing moderate to severe, chronic malignant tumor-related pain. Pain Phys. 2014;17(4):329-343.

78. Baron R, Kern U, Müller M, Dubois C, Falke D, Steigerwald I. Effectiveness and Tolerability of a Moderate Dose of Tapentadol Prolonged Release for Managing Severe, Chronic Low Back Pain with a Neuropathic Component: An Open-label Continuation Arm of a Randomized Phase 3b Study. Pain Pract. Epub 2014 Apr 18.

Therapeutics and Clinical Risk Management

\section{Publish your work in this journal}

Therapeutics and Clinical Risk Management is an international, peerreviewed journal of clinical therapeutics and risk management, focusing on concise rapid reporting of clinical studies in all therapeutic areas, outcomes, safety, and programs for the effective, safe, and sustained use of medicines. This journal is indexed on PubMed Central, CAS,
79. Imanaka K, Tominaga Y, Etropolski M, Ohashi H, Hirose K, Matsumura T. Ready conversion of patients with well-controlled, moderate to severe, chronic malignant tumor-related pain on other opioids to tapentadol extended release. Clin Drug Investig. 2014;34(7):1-11.

80. Niesters M, Proto PL, Aarts L, Sarton EY, Drewes AM, Dahan A. Tapentadol potentiates descending pain inhibition in chronic pain patients with diabetic polyneuropathy. Bri J Anaesth. 2014:aeu056.

81. Vinik AI, Shapiro DY, Rauschkolb C, et al. A randomized-withdrawal, placebo-controlled study evaluating the efficacy and tolerability of tapentadol extended release in patients with chronic, painful diabetic peripheral neuropathy. Diabetes Care. 2014:DC_132291.

82. Erlich DR, Bodine W. Tapentadol (nucynta) for treatment of pain. Am Fam Phys. 2012;85(9):910-911.

83. Biondi DM, Xiang J, Etropolski M, Moskovitz B. Evaluation of blood pressure and heart rate in patients with hypertension who received tapentadol extended release for chronic pain: a post hoc, pooled data analysis. Clinical Drug Investig. 2014;34(8):565-576.

84. Etropolski M, Kuperwasser B, Flügel M, et al. Safety and tolerability of tapentadol extended release in moderate to severe chronic osteoarthritis or low back pain management: pooled analysis of randomized controlled trials. Adv Ther. 2014;31(6):604-620.

85. Wild JE, Grond S, Kuperwasser B, et al. Long-term safety and tolerability of tapentadol extended release for the management of chronic low back pain or osteoarthritis pain. Pain Pract. 2010;10(5):416-427.

86. Bloor M, Paech M, Kaye R. Tramadol in pregnancy and lactation. Int J Obstet Anesth. 2012;21(2):163-167.

87. Tayal G, Grewal A, Mittal R, Bhatia N. Tapentadol: a novel analgesic. J Anaesth Clin Pharmacol. 2009;25(4):463-466.

88. Coluzzi F, Ruggeri M. Clinical and economic evaluation of tapentadol extended release and oxycodone/naloxone extended release in comparison with controlled release oxycodone in musculoskeletal pain. Curr Med Res Opin. 2014;30(6):1139-1151.

89. Crofford LJ, Mease PJ, Simpson SL, et al. Fibromyalgia relapse evaluation and efficacy for durability of meaningful relief (FREEDOM) a 6-month, double-blind, placebo-controlled trial with pregabalin. Pain. 2008;136(3):419-431.

90. Moore RA, Straube S, Derry S, McQuay HJ. Chronic low back pain analgesic studies-A methodological minefield. Pain. 2010;149(3): 431-434.

91. McDermott AM, Toelle TR, Rowbotham DJ, Schaefer CP, Dukes EM. The burden of neuropathic pain: results from a cross-sectional survey. Eur J Pain. 2006;10(2):127-135.

92. Tölle T, Dukes E, Sadosky A. Patient burden of trigeminal neuralgia: results from a cross-sectional survey of health state impairment and treatment patterns in six European countries. Pain Pract. 2006;6(3): $153-160$.

93. Gore M, Brandenburg NA, Hoffman DL, Tai KS, Stacey B. Burden of illness in painful diabetic peripheral neuropathy: the patients' perspectives. J Pain. 2006;7(12):892-900.

94. Argoff CE. The coexistence of neuropathic pain, sleep, and psychiatric disorders: a novel treatment approach. Clin J Pain. 2007;23(1): $15-22$.

95. Obradovic M, Ikenberg R, Hertel N, Antoñanzas F, Gálvez R, Liedgens H. Cost-effectiveness of tapentadol in severe chronic pain in Spain: a cost analysis of data from RCTs. Clin Ther. 2012;34(4):926-943.

\section{Dovepress}

EMBase, Scopus and the Elsevier Bibliographic databases. The manuscript management system is completely online and includes a very quick and fair peer-review system, which is all easy to use. Visit http://www.dovepress.com/testimonials.php to read real quotes from published authors. 